\title{
Location-based Information Fusion for Mobile Navigation
}

\author{
Anna Wu \\ annawu@psu.edu \\ Pennsylvania State University \\ University Park, PA, USA
}

\author{
Xiaolong(Luke) Zhang \\ lzhang@ist.psu.edu \\ Pennsylvania State University \\ University Park, PA, USA
}

\begin{abstract}
Comprehensive yet personalized information for a location is usually desired by mobile users in situ. Traditional navigation systems provide complete static information, such as address, contact, even photos and reviews for a certain place. However, such information does not reflect the real time situation (e.g. popularity/crowdness). Location-based social networks provide opportunity to build social dynamics between the place and potential visitors. In this work, we propose a design by leveraging public online information with users' social network resources to provide real time exploration in novel environments. A mobile application is implemented using Wikipedia, Panoramio, and Foursquare data to provide complete, updated, and trustworthy information. Design highlights and implementation are reported.
\end{abstract}

\section{Author Keywords Navigation, Location-based social} network

ACM Classification Keywords H5.2. [Information interfaces and presentation]: User Interfaces

General Terms Design, Experimentation, Human Factors

\section{INTRODUCTION AND BACKGROUND}

Mobility is an intrinsic part of human development and is essential to modern life. Quickly building spatial awareness of the place and making decisions on where to go is a reasonable demand. Empowered by mobile devices, travelers are able to get comprehensive yet effective information of Point of Interest (POI) while moving. Complete, updated, and trustworthy information is required for trip planning, which must take into consideration of spatiotemporal constraints.

Currently, users have to access multiple resources, such as Wikipedia, contact information and reviews from other people, to get complete information of a point of interest, as the content is confined to separate applications. Navigating between different UI structures is time-consuming and prone to errors [1]. Google Place exemplifies the idea of information fusion by integrating contact information, address, direction, reviews, photos, open hours and official website (when available) into a single application, which provides a one-time shop for such information.

However, such information usually keeps stable, which

Copyright is held by the author/owner(s).

UbiComp'11, September 17-21, 2011, Beijing, China.

ACM 978-1-4503-0630-0/11/09. may not reflect the situation in real time. Location-based social network (LBSN) information provides the dynamics of places that is potentially valuable for a visit. LBSNs leverage users' current location to reveal nearby popular places or friends. Among various LBSN mobile applications proliferated ${ }^{\mathrm{i}}$, Foursquare is one of the widely adopted with 10 million registered users (as of June 2011 ${ }^{\mathrm{ii}}$ ) and 3 million check-ins daily. Users “check-in” to share their location for serendipity, connection, or personal history. Incorporating LBSN information informs navigation design in three ways: a) To some extent, number of check-ins reflects the popularity/crowdness of a certain place at that time (especially true for entertainments venues like bars and restaurants ${ }^{\mathrm{iii}}$ ), which cannot be reflected in traditional tour guide. b) Virtual indicator of physical presence bridges online social network with real world life, which may expose potential interesting places. Pultar and Raubal show that new real world connections are created through LBSNs based on their study of CouchSurfing, a social network for exchange free lodging between travelers cross the world [3]. c) Unlike reviews generated by strangers, comments and visiting history from people in social networks may be more trustworthy and users are more likely to read those updates.

By adding location-based social network data into traditional tour guides, our aim is therefore to provide a). comprehensive static and accumulative information of the place; b). real time information reflecting instant value and cost of the visit; c). content contributions from users' social network. We have developed a functional mobile application prototype to demonstrate our design concept.

\section{THE MOBILE APPLICATION PROTOTYPE}

Figure 1 presents a few key views in the mobile application. The design was based on Nexus One hardware specifications; including a 3.7 inch touch screen. All the views were created for portrait mode except in the camera activity for taking pictures.

After login, users can see a home view with search box, layer selection button (earth icon), POI search button (pin button), and a Google Map view underneath. Like regular

\footnotetext{
i http://techcrunch.com/2008/09/28/the-state-of-location-based-socialnetworking-on-the-iphone/

ii http://techcrunch.com/2011/06/20/foursquare-now-officially-at-10million-

users/?utm_source=feedburner\&utm_medium=feed\&utm_campaign=Feed \%3A+Techcrunch+\%28TechCrunch\%29

iii http://bitsybot.com/foursquare/trends.html\#
} 
navigation tools, users can search for locations by addresses or keywords in the input box. The User can initiate selection from different overlays by clicking the layer selection button, including regular Google Map, Satellite Images, HeatMaps, and Friends Check-ins. HeatMap view takes check-ins from Foursquare of each venue to show popular places near users' current location (Figure 1(a)). Users can select historically accumulation or current checkins to render different overlays. Friends' check-ins are synchronized and updated with the Foursquare server. The POI search button allows users to explore nearby POIs. Category information is labeled by different markers (Figure 1(b)). Further information of a certain place can be examined by clicking on either the friends' check-in item or markers on the map Figure 1(c). Basic information of a location including place name, category, users' tags, address, telephone, email, twitter, Wikipedia page is presented when available (Figure 1(d)). Photo collection from Panoramio about this location is shown in the Gallery view. User can take their own pictures and upload them to our application server, which are automatically added to the Gallery collection. Users can also add interested places to a visit list, similar to adding goods into shopping carts. The visiting list can be modified by adding or removing certain places. An optimized route using sales-man algorithm can be generated covering all the places planned from user's current location, or other user defined locations.
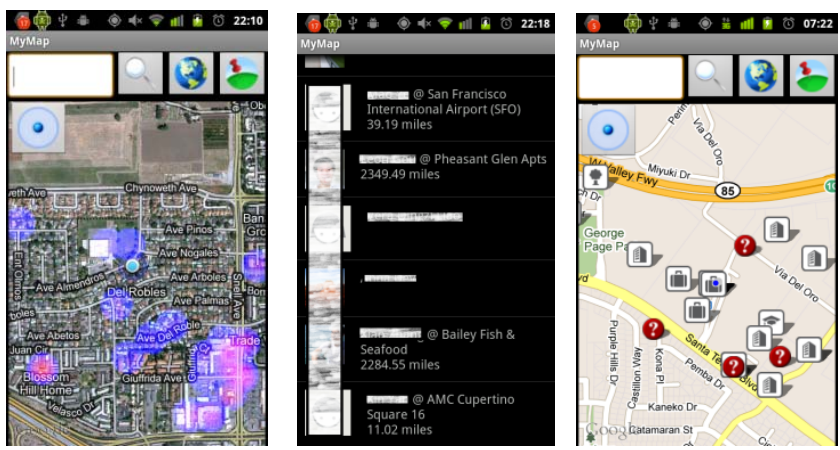

(a) HeatMap

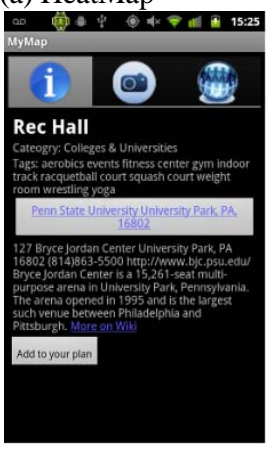

(b) Friends' checkins

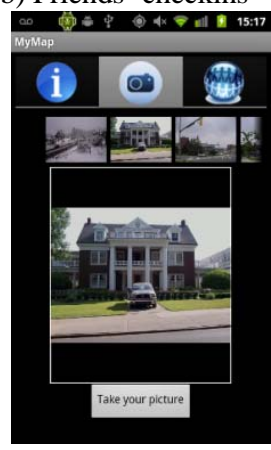

c) Nearby POIs

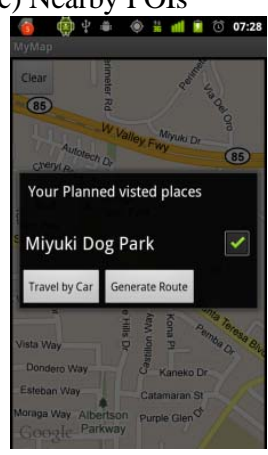

(d) Info. page

(e) Gallery

(f) Route planning

Figure 1. Example Views.

The application is written in Java using the Google SDK API level 8. Transition of different views is implemented through Android mechanism for launching activities, aka
Intents. Venue information and users' check-ins are accessed from REST-based Foursquare API v2 i. HTTP response as JSON object is extracted and matched into java models for each place. Wikipedia information is extracted through Wikimapia API ii based on the proximity and keywords match. Panoramio Data API is applied to retrieve photos nearby ${ }^{\text {iii }}$. User-taken photos are uploaded and stored in a Tomcat server with metadata extracted and stored in MySQL. The application is able to synchronize users' own content created (e.g. photos) and content shared by their contacts with services.

\section{DISCUSSION AND CONCLUSION}

Two assumptions of the targeted users are inherently imposed for the application:

a) Users want to explore the environments and build the spatial sense by visiting multiple places, instead of simply "get me to location $\mathrm{X}$ ".

b) Users have social network friends who check out places and post their check-ins and comments online. Location privacy is seriously debated and protective methods are proposed by both researchers [4] and public media[2], we assume users of our application are willing to share their positions.

Integrating geographical information from different webservices is challenging due to the lack of universal identification system. The identifier and latitude/longitude of a certain geographical ontology in one service might be different in another service. Unify references across databases is the key issue to improve data quality for location-based information fusion.

\section{REFERENCES}

1. Cui, Y., Oulasvirta, A., Ma, L. Event Perception in Mobile Interaction: Toward Better Navigation History Design on Mobile Devices. International Journal of Human-Computer Interaction, 27, 5 (2011), 413-435.

2. Perez, S. Location-Based Social Networks: Delightful, Dangerous or Somewhere in Between? ReadWriteWeb, 2010.

http://www.readwriteweb.com/archives/locationbased_social_networks_delightful_dangerous.php.

3. Pultar, E. and Raubal, M. A case for space: physical and virtual location requirements in the CouchSurfing social network. Proc of LBSN'09, (2009).

4. Toch, E., Cranshaw, J., Hankes-Drielsma, P., Springfield, J., and Kelley, P.G. Locaccino: a privacy-centric location sharing application. Proc of Ubicomp'10, (2010).

\footnotetext{
i https://developer.foursquare.com/

ii http://wikimapia.org/api/

iii http://www.panoramio.com/api/data/api.html
} 